\title{
Hybrid minimally invasive esophagectomy: a goal or a step?
}

\author{
Guillaume Piessen $^{1,2}$, Clarisse Eveno ${ }^{1,2}$, Thibault Voron ${ }^{1}$ \\ ${ }^{1}$ Department of Digestive and Oncological Surgery, Claude Huriez University Hospital, Lille, France; ${ }^{2}$ University Lille, UMR-S $1172-C A N T H E R$ \\ Laboratory "Cancer Heterogeneity, Plasticity and Resistance to Therapies", Lille, France \\ Correspondence to: Guillaume Piessen, MD, PhD. Department of Digestive and Oncological Surgery, University Hospital Claude Huriez, CHRU- \\ Place de Verdun 59037 Lille, France. Email: guillaume.piessen@chru-lille.fr. \\ Provenance and Peer Review: This article was commissioned and reviewed by the Section Editor Lei Deng, MD (Jacobi Medical Center, Albert \\ Einstein College of Medicine, Bronx, NY, USA). \\ Response to: Peng JS, Hochwald SN. Minimally invasive esophagectomy-standard of care. J Thorac Dis 2019;11:S1387-8. \\ Pimiento JM, Fontaine JP. Minimally invasive transthoracic esophagectomy: pushing the boundaries to improve surgical outcomes. J Thorac Dis \\ 2019;11:S1336-8.
}

Submitted Feb 09, 2020. Accepted for publication May 30, 2020.

doi: $10.21037 /$ jtd-20-821

View this article at: http://dx.doi.org/10.21037/jtd-20-821

We thank the editor for giving us the opportunity to answer to two commentaries regarding our recent publication (1-3). As underlined by Peng et al. (2), minimally invasive esophagectomies (MIE) for oncological indications have been increasing over time in the different continents, and now becomes a well evaluated procedure with 3 randomized control trials recently published comparing totally MIE (TMIE), TMIE with a robotic thoracic phase and hybrid MIE (HMIE) (1,4,5). A recent study reported an international high volume esophagectomy centers experience on 2704 esophagectomies between January 2015 and December 2016. Surgical approach involved MIE in only $47.9 \%$ of cases. Among them, $48.7 \%$ were done with a totally minimally invasive approach. The rest of the procedures $(51.3 \%)$ were hybrid with mostly a minimally invasive abdominal phase and an open thoracic approach similar to the MIRO trial (40.2\%) (6). This suggests that the road is long before a full adoption of TMIE and HMIE even in expert centers. HMIE is more a step than a goal and a complete adoption TMIE would probably be a major progress in the field.

\section{Short term results}

We acknowledge with Peng that definitions used in the TIME trial and in the MIRO trial were different (2). Of note the primary outcome in the TIME trial was only postoperative pulmonary infection defined as "clinical manifestation of pneumonia or bronchopneumonia confirmed by thoracic radiographs or CT scan (assessed by independent radiologists) and a positive sputum culture, within the first 2 weeks of surgery and during the whole stay in hospital" which are in fact very restrictive (4). Interestingly, the observed rate in the open and TMIE group was quite high (34\% and $12 \%$, respectively). This significant difference may be explained by the difference of technic of ventilations between the two arms as underlined by our team in a letter to the Lancet (7). Of note the observed rates of pneumonia were even higher in the ROBOT trial (55\% vs. 28\%) (5). In the MIRO trial, the definition of pneumonia was an "alveolo-interstitial radiologic infiltration with the presence of at least two of the following criteria: purulent sputum, temperature $>38.5^{\circ} \mathrm{C}$ or $<35^{\circ} \mathrm{C}$ or leukocytes $>10,000 / \mathrm{mm}^{3}$ or $<1,500 / \mathrm{mm}^{3}$ ". The 30 -day rates were more reasonable in both groups $(16.5 \%$ in the open $v s .12 .8 \%$ in the hybrid group). The definition of major pulmonary complications was much larger including major bronchial sputum, pneumonia, respiratory failure, and acute respiratory distress syndrome (ARDS) within 30 days than open surgery and reached $30 \%$ in the open group versus $18 \%$ in the hybrid group within 30 days (odds ratio, $0.50 ; 95 \%$ CI, 0.26 to 0.96 ) (1).

The problem of anastomotic site is of importance with as noted by Peng et al. (2). TMIE with intra-thoracic anastomosis is a challenging approach with a learning curve up to 119 cases and a higher risk of anastomotic 
leakage at least in the first cases (8). Modifications of the surgical technique may consequently have been initially required such a performance of a cervical anastomosis instead of an intra-thoracic anastomosis. This is what we observed in the TIME (two third of patients undergoing cervical anastomosis) and in the ROBOT trial (all cervical anastomosis) (4,5). A cervical anastomotic site has been associated with a potential higher risk of post operative mortality and more importantly in the context of MIE of significant morbidity with an increased median Comprehensive Complication Index (CCI), and should consequently be avoided unless the tumor location importantly it $(9,10)$.

We agree with Piemento et al. (3) that the length of stay after esophagectomy may be more explained by the changes in gastrointestinal physiology and the extend of dissection than by the length of incisions. In the MIRO trial, the median length of hospital stay was similar between groups, which highlights that this outcome is affected by several aspects of the patient treatment pathway, and perhaps most importantly enhanced recovery protocols, which were strictly adhered to in both study arms.

\section{Long term results}

In both Time and Robot trial, oncological outcomes were comparable between the two groups $(5,11)$. In the MIRO trial, there were no significant differences in 3-year overall and disease-free survival with however-a clear trend in favor of the hybrid approach (hazard ratio for death of 0.67 (95\% CI, 0.44 to 1.01) for overall survival) (1). Long term results are being analysed and will probably give an interesting insight on this tendency. Of note, a recent metaanalysis combining hybrid and totally miniinvasive esophagectomy confirmed that MIE was associated with an $18 \%$ lower 5 -year all-cause mortality than open approach (HR 0.82, 95\% CI 0.76-0.88). The meta-regression analysis showed no confounding (12). One can hypothesize that MIE is associated with fewer and less serious complications. This may permit retainment of immuno-competence in a higher number of patients, with consequently avoiding or delaying tumor recurrence and resulting death.

\section{Quality of life}

As discussed by Peng et al., TMIE improved quality of life in both TIME trial in the post operative period and after 1 year especially regarding physical health and pain and ROBOT trial (at discharge and at 6 weeks) $(2,4,5,13)$. Results were more conflicting for HMIE in the MIRO trial (14). We found that esophagectomy had substantial effects upon short-term HRQOL. These effects for some specific parameters were however, reduced with HMIE, with persistent differences up to 2 years, and maybe mediated by a reduction in postoperative complications. However after 3 years, no statistical significant differences were found between HMIE and open approach in changes from baseline health related quality of life.

\section{Learning curve}

As underlined by Peng et al., TMIE with either a conventional or a robotic approach have significant learning curves. Consequently, successful adoption requires investment of resources and time (2). A volume threshold of 25 laparoscopic phases was chosen for entry into the MIRO trial (1). This volume threshold was selected through a Delphi consensus process among participating centers. This threshold has been also described as being suitable for MIE in a nationwide study (15). HMIE is attractive because it doesn't modify the confection of esophagogastric anastomosis, which is a pivotal moment during the surgery conditioning post-operative outcomes. We acknowledge with Pimiento et al. that adoption of a new surgical approach should be implemented in high-volume centers by high volume surgeons to minimize the learning curve and improve outcomes (3). Definition of "quality benchmarks" through expert centers experiences should be encouraged.

In conclusion, MIE, either HMIE or TMIE is associated with improvement in short term outcomes, quality of life and potentially long term outcomes and should now be the standard in oesophagectomy. HMIE and TMIE offer a similar magnitude of benefit on morbidity (OR 0.31 vs. 0.30). Comparison between TMIE and HMIE is of scientific interest but expected differences are small and would necessitate large numbers of patients to be included. More than put in opposition the two techniques, having them both to be used according to : patient profile, tumor extension, center/surgeon expertise and patient's desire seems to be much appropriate. Moreover, the advent of robotic surgery will surely overcome technical difficulties related to perform an intrathoracic anastomosis and further allow improvement of postoperative outcomes after TMIE. HMIE and TMIE should be considered the standard of care for patients undergoing esophagectomy and can be completed as planned in the vast majority of cases. 


\section{Acknowledgments}

Funding: None.

\section{Footnote}

Conflicts of Interest: All authors have completed the ICMJE uniform disclosure form (available at http://dx.doi. org/10.21037/jtd-20-821). GP reports personal fees from Amgen, personal fees from Roche, personal fees from Medtronic, outside the submitted work. The other authors have no conflicts of interest to declare.

Ethical Statement: The authors are accountable for all aspects of the work in ensuring that questions related to the accuracy or integrity of any part of the work are appropriately investigated and resolved.

Open Access Statement: This is an Open Access article distributed in accordance with the Creative Commons Attribution-NonCommercial-NoDerivs 4.0 International License (CC BY-NC-ND 4.0), which permits the noncommercial replication and distribution of the article with the strict proviso that no changes or edits are made and the original work is properly cited (including links to both the formal publication through the relevant DOI and the license). See: https://creativecommons.org/licenses/by-nc-nd/4.0/.

\section{References}

1. Mariette C, Markar SR, Dabakuyo-Yonli TS, et al. Hybrid Minimally Invasive Esophagectomy for Esophageal Cancer. N Engl J Med 2019;380:152-62.

2. Peng JS, Hochwald SN. Minimally invasive esophagectomy-standard of care. J Thorac Dis 2019;11:S1387-8.

3. Pimiento JM, Fontaine JP. Minimally invasive transthoracic esophagectomy: pushing the boundaries to improve surgical outcomes. J Thorac Dis 2019;11:S1336-8.

4. Biere SS, van Berge Henegouwen MI, Maas KW, et al. Minimally invasive versus open oesophagectomy for patients with oesophageal cancer: a multicentre, open-label, randomised controlled trial. Lancet 2012;379:1887-92.

5. van der Sluis PC, van der Horst S, May AM, et al. Robot-assisted Minimally Invasive Thoracolaparoscopic Esophagectomy Versus Open Transthoracic Esophagectomy for Resectable Esophageal Cancer: A
Randomized Controlled Trial. Ann Surg 2019;269:621-30.

6. Low DE, Kuppusamy MK, Alderson D, et al. Benchmarking Complications Associated with Esophagectomy. Ann Surg 2019;269:291-8.

7. Mariette C, Robb WB. Minimally invasive versus open oesophagectomy for oesophageal cancer. Lancet 2012;380:883-6.

8. van Workum F, Stenstra MHBC, Berkelmans GHK, et al. Learning Curve and Associated Morbidity of Minimally Invasive Esophagectomy. A Retrospective Multicenter Study. Ann Surg 2019;269:88-94.

9. Degisors S, Pasquer A, Renaud F, et al. Are Thoracotomy and/or Intrathoracic Anastomosis Still Predictors of Postoperative Mortality After Esophageal Cancer Surgery?: A Nationwide Study. Ann Surg 2017;266:854-62.

10. Schröder W, Raptis DA, Schmidt HM, et al. Anastomotic Techniques and Associated Morbidity in Total Minimally Invasive Transthoracic Esophagectomy: Results From the EsoBenchmark Database. Ann Surg 2019;270:820-6.

11. Straatman J, van der Wielen N, Cuesta MA, et al. Minimally Invasive Versus Open Esophageal Resection: Three-year Follow-up of the Previously Reported Randomized Controlled Trial: the TIME Trial. Ann Surg 2017;266:232-6.

12. Gottlieb-Vedi E, Kauppila JH, Malietzis G, et al. Longterm Survival in Esophageal Cancer After Minimally Invasive Compared to Open Esophagectomy: A Systematic Review and Meta-analysis. Ann Surg 2019;270:1005-17.

13. Maas KW, Cuesta MA, van Berge Henegouwen MI, et al. Quality of Life and Late Complications After Minimally Invasive Compared to Open Esophagectomy: Results of a Randomized Trial. World J Surg 2015;39:1986-93.

14. Mariette C, Markar S, Dabakuyo-Yonli TS, et al. Healthrelated Quality of Life Following Hybrid Minimally Invasive Versus Open Esophagectomy for Patients With Esophageal Cancer, Analysis of a Multicenter, Open-label, Randomized Phase III Controlled Trial: The MIRO Trial. Ann Surg 2020;271:1023-9.

15. Mackenzie H, Markar SR, Askari A, et al. National proficiency-gain curves for minimally invasive gastrointestinal cancer surgery. Br J Surg 2016;103:88-96.

Cite this article as: Piessen G, Eveno C, Voron T. Hybrid minimally invasive esophagectomy: a goal or a step? J Thorac Dis 2020;12(7):3908-3910. doi: 10.21037/jtd-20-821 